\title{
Middle grade students reasoning about temporary magnetism
}

\author{
Tamara G. Young \\ Physics \& Astronomy, University of Utah, 115 S. 1400 E. 201 JFB, SLC, UT 84112 \\ Lauren A. Barth-Cohen \\ Educational Psychology, University of Utah, 1721 Campus Center Dr. SAEC 3220, SLC, UT 84112 \\ Physics \& Astronomy, University of Utah, 115 S. 1400 E. 201 JFB, SLC, UT 84112 \\ Sarah K. Braden and Sara Gailey \\ School of Teacher Education and Leadership, Utah State University, 2805 Old Main Hill, Logan, UT 84322 \\ Magnetism is a significant topic in physics and student reasoning about magnetism is of interest to PER. \\ Temporary magnetism is encountered during scientific inquiry, and pervasive in school physics, despite it typ- \\ ically not being an explicit part of the curriculum. We examine how middle grade students reason with this \\ complex topic, and findings demonstrate that students' reasoning about different facets of this topic reflect that \\ complexity.
}




\section{INTRODUCTION}

While temporary magnetism is a pervasive topic in K-12 education, it is also one that is not well studied. Many studies that focus on how older students reason about magnetism in general [1-6] do not include reasoning about temporary magnetism. Here we examine how students reason about temporary magnetism. This qualitative analysis comes from a 7 th grade classroom where students were engaged in a Next Generation Science Standards, NGSS [7], aligned curriculum on modeling magnetism. We found that students reasoned about different facets of temporary magnetism: that objects can become temporarily magnetized, that temporary magnetism results from unseen elements, and they wrestled with the nature and implications of being temporarily magnetized. Numerous factors influence temporary magnetism including variations in the time scales of magnetization. These factors may lead students to make conflicting observations. In spite of this, the students demonstrate sophisticated reasoning which suggests that temporary magnetism is a topic that deserves more focus than the field of PER has traditionally realized.

Temporary magnetism is often overlooked in magnetism curricula. For example, temporary magnetism is not addressed in most undergraduate physics textbooks [8, 9]. While magnetism is embedded in the NGSS Disciplinary Core Ideas related to forces [7], temporary magnetism is not specifically included. However, temporary magnetism is pervasive in school physics. That is, students who are engaging in inquiry-based learning involving magnetism will undoubtedly encounter temporary magnetism. For example, physics students are often asked to temporarily magnetize a nail or similar to demonstrate that ferrous objects can become magnetized. In addition, ferromagnetic objects left in contact with external magnetic fields may become temporarily magnetized and permanent magnets stored incorrectly become unmagnetized. Importantly, how a student reasons about temporary magnetism may influence their understanding of both permanent magnetism and electromagnetism, but yet temporary magnetism is traditionally unexplored in PER. Research has focused on other important aspects such as electromagnetic induction [1] and vector fields [2, 3]. However, as temporary magnetism is commonly encountered during inquiry-based lessons on magnetism, the students' experience with temporary magnetism may be a confounding factor in how the students understand magnetism.

A significant body of research has explored students' understanding of concepts in electricity and magnetism within various force concept inventories $[10,11]$. While these studies provide valuable resources about student conceptions, they do not provide insight into how students reason about magnetism, and they overlook temporary magnetism.

Other research has approached magnetism from a more qualitative viewpoint in order to examine student reasoning about magnetism [1]. However, much of this research [1-6] is focused on undergraduate and high school students. Whereas less research is focused on younger grades, despite students in those lower grades commonly encountering temporary magnetism in their instruction. While we have seen that younger students are capable of reasoning that reflects the more sophisticated reasoning of high school and undergraduate students [12], it remains instructive to examine the reasoning processes of younger students. For example, conceptual systems developed in earlier grades are often carried into undergraduate physics courses. In addition, problem solving ability often acts as a confounding variable in examining students' conceptual understanding in high school and beyond $[4,6]$.

Therefore we see that student reasoning about temporary magnetism is a topic of interest in PER. However, we also see that student reasoning about temporary magnetism is largely unexplored and that much of the research on student reasoning about magnetism is focused on high school and undergraduate students. This paper attempts to address these gaps by asking the research question, how do middle grade students reason about temporary magnetism?

\section{METHODS}

The research setting was a 7 th grade science class at a charter school in the Intermountain West. The population of the students mirrored the population of the district which was $46.8 \%$ White and $53.2 \%$ racial or ethnic minority. Researchers collaborated with a 7 th grade science teacher to develop and implement an NGSS aligned curriculum modeling magnetism. The teacher held a Ph.D. in biology and had a prior professional relationship with the researchers. Twentynine students agreed to participate in the research study. The theoretical framework we use for the analysis is a resource framework, in which we assume that students have productive knowledge resources, including ways of thinking, existing experiences, prior knowledge, etc. that can be leveraged for future learning[13].

The curriculum is as follows: 1) The students engaged with the initial phenomena, a floating paperclip. 2) The students created an initial mechanistic model of the floating paperclip. 3) The students explored other properties of magnetism at 8 stations in small groups. 4) The students revised their initial individual models. 5) The students shared their models with their small groups. 6) The students worked in their small groups to develop a consensus model. 7) The groups presented the consensus model to the full class. Finally, 8) the students completed an individual assessment. The sequence took approximately 2 weeks of instruction in which the researchers were participant observers in the classroom. This sequence was informed by Passmore, Schwarz, and Mankowski [14] and Kenyon, Schwarz, and Hug [15]. For more details about the instructional sequence see Braden, Barth-Cohen, Gailey and Young [16].

Data was collected during all parts of the instructional sequence and included: researcher field notes, audio recordings of all groups, video recordings of some stations, audio and video recordings of final presentations, final group models, 
and individual assessments. From the researcher notes, we determined that the final presentations had the richest data, and so focused the analysis on this data. During the final presentations the teacher led a discussion in which all students were required to participate. The presenting groups described their models and cited evidence to support their models. The teacher scaffolded the conversation by instructing students to be prepared to comment on the presenters' model, either by agreeing with the model and providing evidence to support the model, asking a question about the model, or by disagreeing with the model and providing evidence to support their disagreement. This full-class discussion took over an hour and ranged over a variety of topics, such as the shape and strength of the magnetic fields, the arrangements of microscopic elements in the magnet and paperclip, the polarity of the magnets and paperclips, and others.

Once we had decided to focus on the final presentations for our analysis, we transcribed that data using conversation analysis conventions [17]. Next we underwent an iterative process of discussing the conversations to establish internal consistency [18]. During this process, we observed that temporary magnetism was an important topic in the discussion; it was mentioned by each of the presenting groups and was a topic of primacy in the first and the fourth/final presentations. Next we wrote a summary explaining temporary magnetism that included a brief discussion hypothesizing why students would struggle with this. This led us to recognize how the students' reasoning about temporary magnetism affected their overall understanding of magnetism.

Having recognized the importance of students' reasoning about temporary magnetism, we enacted a subsequent analysis of ways the students struggled productively with temporary magnetism. Here we used open coding to identify themes at the turn of talk level [19]. First we selected each instance when a student discussed the magnetic nature of the paperclip resulting in 30 statements made by 14 students. The statements come from all 4 presentations as well as 6 follow-up questions and conversations. Once we had selected relevant utterances, we identified and clustered the main ideas. This was an iterative process in which some of the initial concepts were folded into larger themes, which we present in this paper. As this was a teacher led discussion, we do not claim to capture the full range of understanding of all students. Still, the analysis revealed several facets of how students reasoned about the nature of temporary magnetism.

\section{RESULTS}

Temporary magnetism is a complex topic, and students' reasoning about it reflects that complexity. Overall, we found that students reason about different facets of the topic, including hypotheses on what happens to a ferromagnetic item in an external magnetic field and how that object behaves when the external magnetic field is removed or changed. Intertwined in these discussions, students wrestled with temporary mag- netism. In particular, they argued whether the ferromagnetic object becomes a temporary magnet and what it means to be a temporary magnet. Part of this complexity is a result of the sometimes conflicting evidence that is used to support their reasoning [12]. In addition, they are reasoning about a micro level that is too small to be seen, which is difficult for students [20].

Here we present different facets of how students reason about temporary magnetism. The ideas are presented in the following order based on increasing complexity. First, we present data on how students describe the paperclip becoming magnetized. Next, we show how students generate possible explanations for why the paperclip sticks to the magnet. Finally, we present data about the students discussing what it means to be a temporary magnet, and what happens to the paperclip when the external magnetic field is changed. These ideas are interconnected and often interwoven cyclically through the discussion, although there is some temporal progression. The student statements presented in this section are not in temporal order, rather they are discrete examples of the various ways the students discussed temporary magnetism.

\section{A. Magnetization}

First, widespread throughout the class, was the notion that the paperclip becomes magnetized when touching or near the permanent magnet. In addition to claiming that the paperclip becomes a temporary magnet, students provided evidence to support this claim.

"When the permanent magnet touches the paper clip, it makes the paperclip turn into a temporary magnet."

"[The paperclip] becomes a magnet cause it can pick up different objects."

In the second example, the student cites evidence that the paperclip has become a magnet because the paperclip can pick up different objects. This observation could have been made at one of several different stations in which the students interacted with magnets and paperclips. The idea that the paperclip became a temporary magnet was widespread in the classroom and expressed by different groups during the discussion.

\section{B. Unseen elements}

Another facet that emerged were two mechanisms that explained what was causing the paperclip to be attracted to the magnet. Students argued that the magnetic field encloses the paperclip. Students also argued that the "molecules" in the paperclip near the magnet are more "organized," or "aligned" with the magnetic field of the magnet. The following quote illustrates the first explanation. 
"The magnetic fields surrounding the paperclip, attracts the molecules"

Additionally, the next quotes illustrate the second explanation.

"With the paperclip, if it's in the magnetic field, it [the molecules] shifts. How it, it shifts so that it, like, more organized."

"The molecules of both are being aligned. \#\#\# when it's touching."

Both of these explanations are reasonable and potentially productive given their inclusion of mechanistic reasoning [21] with an emphasis on the actions of unseen elements.

Often we see that these ideas build on each other; the external magnetic field pulls on the "molecules", it causes the "molecules" to become more organized or to align. However, the students do not necessarily agree with how the external magnetic field interacts with the "molecules" of the paperclip, resulting in disagreement about temporary magnetism. An example of this occurs when a student argues that the paperclip is not a temporary magnet, in spite of being able to pick up other things. Instead he says that "The magnet pull, will ahh, almost goes through the objects [which causes them to] attract to another." In other words the external magnetic field is causing that attraction, not the paperclip. In Sec. III C the students continue the disagreement about temporary magnetism.

Here we have shown two ways that students provide a mechanism for the attraction of the paperclip to the magnet: the magnetic field, and the alignment of the "molecules." These ideas can either be complementary or contradictory.

\section{The nature of temporary magnets}

We also see that the students disagree with the nature of temporary magnetism. The students disagree about two facets of the temporary magnets: does the paperclip have poles? and what happens when you change the external magnetic field?

Some students argue that when the paperclip is within the external magnetic field, the "molecules" are aligned with this field, resulting in the paperclip becoming a temporary magnet that has a north and a south pole, just like a permanent magnet. For example, one student argued

"It's [the molecules] lined up, south facing to the south and north to the north and then they are also lined up with south being down and north being up."

While a different student explained:

"I would say that, it [the paperclip] has a north and south pole, but only when another magnet is on it."
Other students argue that the paperclip cannot have a north and south pole. Some students provide evidence that the paperclip is always attracted to permanent magnets, but permanent magnets both attract and repel other permanent magnets. One student states:

"[The] paperclip always sticks to the magnet even though sometimes they [magnets] repel each other."

This student goes on to say that this is because of the magnetic "flow" of the external magnet. This implies that the attraction of the paperclip is not because the "molecules" align with the external magnetic field, but that the external magnetic field pulls on these "molecules." This highlights the difference between the "alignment" of the molecules and "pull" on the molecules previously mentioned in Sec. III B. Here we see the disagreement the students have about whether or not a temporary magnet has a north and south pole.

Finally, the students discuss how the temporary magnet would behave if you changed the external magnetic field. Some students argue that the "molecules" can change sides, others argue that they would not "just flip back and forth." Another group of students wonder if the entire paperclip might flip over.

"I think that the paperclips can change which side is north and which side is south. The second one that is, will be put on, will just switch to the whichever is attractive."

"I disagree with, that they [the paperclip] can switch poles."

"Would the paperclip, be like, completely flip itself around?"

These quotes highlight three different ways the students reasoned about what might happen to the paperclip when the external magnetic field was changed: the paperclip changes poles, does not change poles, or the paperclip flips itself over.

\section{DISCUSSION}

In these conversations we see many ways in which the students think about the concept of temporary magnetism. We see that the students are deeply wrestling with what happens at the microscopic level, which is hard, as it is unseen, but also is essential for explaining temporary magnetism. The students argued whether or not the paperclip became a temporary magnet. Students stated that the paperclip is enclosed in the magnetic field, which may or may not result in a realignment of the "molecules" such that the paperclip "gained poles." When considering what would happen if the external magnetic field were to change, the students proposed different interpretations regarding the behavior of the microscopic and macroscopic elements of the magnet. Often the students used evidence to support their contradictory claims. Often the 
explanations fit the evidence and explain additional observations, but differ in ways from the canonical understanding of temporary magnetism. Sometimes correct explanations are offered without evidence, which then fails to convince other students. Many of these ideas, while not "correct" are fruitful and show that the students are thinking in ways that are sophisticated because they are able to support their claims with evidence, make connections between the macroscopic and microscopic levels, and craft explanations based on unseen elements.

There are many reasons that students might struggle with temporary magnetism: One confounding factor is that student understanding of temporary magnetism is dependent on their understanding of the microscopic elements. Connecting the microscopic elements with the macroscopic phenomena is a known difficulty [20]. Consider a model in which the microscopic elements within the paperclip are represented as dipoles that align with the external magnetic field when in the magnetic field and align randomly when not in the magnetic field. Such a model would allow a student to make correct predictions about what those domains would do if the magnetic field were reversed. Conversely, a model that did not include those dipole elements might not.

Another reason that students may struggle with temporary magnetism is the complex nature of temporary magnetism itself; there are factors that influence both the timescales and the amount of magnetization of a temporary magnet. When something is temporarily magnetized, enough of the domains align with the external magnetic field that the temporarily magnetized item becomes a magnet. However, as this is a temporary property, it is often difficult to observe, which presents as a reasoning and instructional challenge. A paperclip might become a magnet within an external magnetic field, but that magnetism lasts for seconds when the external field is removed, and for a fraction of a second when exposed to a different external field. The variability in these timescales may have led to students making conflicting observations. Similarly, there are many factors that influence how much the paperclip may become magnetized (e.g. strength of the external magnet, the magnetic susceptibility of the paperclip, etc.) which may also have contributed to the students to making conflicting observations about the behavior of the paperclip.

In may ways, this study contributes to the body of knowledge on how students think about magnetism. For example, in Jelicic, Planinic, \& Planinsic [1], the researchers observe that difficulty in differentiating between monopoles and dipoles leads to difficulties in understanding magnetic induction. This research also recognizes the importance of microscopic dipole elements. Another way this contributes to the larger body of research is here we postulate that students may make conflicting observations due to the time dependent nature of temporary magnetism, by addressing these issues students may develop intuitions about delayed potentials that could be leveraged when students learn about Maxwellian models of electromagnetism [2].

Although temporary magnetism is typically not an explicit part of school curricula, temporary magnetism is encountered in scientific inquiry, and is pervasive in school physics. For this reason, research in how students reason with temporary magnetism is relevant to PER. In this paper, we have shown many complex and sophisticated ways that students reason about temporary magnetism. However, many questions are as yet unexplored regarding why students reason in these ways and the impacts of this on future learning. Therefore, additional research on this topic is needed.

\section{ACKNOWLEDGMENTS}

We thank the students and teachers who helped contribute to this research by opening up their classroom and embracing this collaboration. We would like to thank Taylor Dexter for assisting with data collection.
[1] K. Jelicic, M. Planinic, and G. Planinsic, Analyzing high school students' reasoning about electromagnetic induction, Phys. Rev. Phys. Educ. Res. 13, 010112 (2017).

[2] K. Zuza, P. van Kampen, M. De Cock, T. Kelly, and J. Guisasola, Introductory university physics students' understanding of some key characteristics of classical theory of the electromagnetic field, Phys. Rev. Phys. Educ. Res. 14, 020117 (2018).

[3] L. Bollen, P. van Kampen, and M. De Cock, Students' difficulties with vector calculus in electrodynamics, Phys. Rev. Spec. Top. Phys. Educ. Res. 11, 020129 (2015).

[4] E. Bagno and B.-S. Eylon, From problem solving to a knowledge structure: An example from the domain of electromagnetism, Am. J. Phys. 65, 726 (1997).

[5] T. M. Scaife and A. F. Heckler, Interference between electric and magnetic concepts in introductory physics, Phys. Rev. Spec. Top. Phys. Educ. Res. 7, 010104 (2011).
[6] E. R. Savelsbergh, T. de Jong, and M. G. Ferguson-Hessler, Choosing the right solution approach: The crucial role of situational knowledge in electricity and magnetism, Phys. Rev. Spec. Top. Phys. Educ. Res. 7, 010103 (2011).

[7] NGSS Lead States, Next generation science standards: For states, by states (National Academies Press, Washington, DC, 2013).

[8] D. Halliday, R. Resnick, and J. Walker, Fundamentals of physics (John Wiley \& Sons, 2013).

[9] R. A. Serway and J. W. Jewett, Physics for scientists and engineers (Cengage learning, 2018).

[10] e.g. D. P. Maloney, T. L. O'Kuma, C. J. Hieggelke, and A. Van Heuvelen, Surveying students' conceptual knowledge of electricity and magnetism, Am. J. Phys. 69, S12 (2001).

[11] e.g. M. W. McColgan, R. A. Finn, D. L. Broder, and G. E. Hassel, Assessing students' conceptual knowledge of electric- 
ity and magnetism, Phys. Rev. Phys. Educ. Res. 13, 020121 (2017).

[12] L. Barth-Cohen, S. K. Braden, T. G. Young, and S. Gailey, Reasoning with evidence while modeling: Successes at the middle school level, Phys. Rev. Phys. Educ. Res. 17, 020106 (2021).

[13] D. Hammer and E. van Zee, The beginnings of scientific reasoning, in Seeing the science in children's thinking: Case studies of student inquiry in physical science (New York: Heinemann, 2006) pp. 13-37.

[14] C. V. Schwarz, C. Passmore, and B. J. Reiser, Helping students make sense of the world using next generation science and engineering practices (NSTA Press, 2017).

[15] L. Kenyon, C. Schwarz, and B. Hug, The benefits of scientific modeling, Sci. Child. 46, 40 (2008).

[16] S. K. Braden, L. Barth-Cohen, S. Gailey, and T. G. Young, Modeling magnetism with the floating paper clip: Supporting diverse learners with visual and linguistic scaffolds, Sci. Scope
44 (2021).

[17] J. DuBois and S. Cumming, Discourse transcription, in Santa Barbara papers in linguistics, edited by Vol. 4S. SchuetzeCoburn and D. Paolino (University of California, Santa Barbara, Department of Linguistics, 1992).

[18] R. E. Stake, The art of case study research (Sage, 1995).

[19] M. Savin-Baden and C. Howell-Major, Qualititative research: The essential guide to theory and practice (Routledge, 2013).

[20] P. Sengupta and U. Wilensky, Learning electricity with NIELS: Thinking with electrons and thinking in levels, Int. J. Comp. Math. Learn. 14, 21 (2009).

[21] R. S. Russ, R. E. Scherr, D. Hammer, and J. Mikeska, Recognizing mechanistic reasoning in student scientific inquiry: A framework for discourse analysis developed from philosophy of science, Sci. Educ. 92, 499 (2008). 